\title{
DISTRIBUCIÓN Y CONSERVACIÓN DE LAS POBLACIONES NATURALES DE PINUS GREGGII
}

\author{
C. Ramírez-Herrera, J. J. Vargas-Hernández y J. López-Upton \\ Programa Forestal, Colegio de Postgraduados \\ km. 36.5 Carr. México-Texcoco, 56230. México \\ correo electrónico: vargashj@colpos.mx, uptonj@colpos.mx
}

\section{RESUMEN}

P. greggii Engelm. es endémico del este de México, con amplia importancia ecológica y económica. Su área de distribución natural se encuentra entre los $20^{\circ} 13^{\prime}$ y los $25^{\circ} 29^{\prime}$ de latitud N, con una discontinuidad de $360 \mathrm{~km}$ en la parte central. Las poblaciones del norte se conocen como $P$. greggii var. greggii y las del sur como $P$. greggii var. australis. El intervalo altitudinal de la especie es de 1,400 a 2,613 m s.n.m. Los rodales del sur se encuentran a menor elevación, con mayor temperatura media y precipitación anual $( \pm 1,720 \mathrm{~m}$, $\left.\pm 17.5^{\circ} \mathrm{C} \mathrm{y} \pm 1,370 \mathrm{~mm}\right)$ que las poblaciones de la región norte $\left( \pm 2,450 \mathrm{~m}, \pm 13^{\circ} \mathrm{C} \mathrm{y} \pm 640 \mathrm{~mm}\right)$. Se muestrearon veinte poblaciones naturales de la especie, encontrándose considerables diferencias entre ellas en cuanto a la superficie que cubren y las especies arbóreas asociadas. En la porción sur se registraron plantas típicas de comunidades templado-cálidas, mientras que en el norte abundaron los árboles de climas frescos y semiáridos, aunque Juniperus flaccida y Quercus spp. son comunes en las dos regiones. En general las poblaciones en los extremos de la distribución natural presentan menor tamaño y menor densidad de arbolado maduro y regeneración. En la mayoría de las poblaciones de $P$. greggii el impacto humano es notable a causa del aprovechamientos de madera y actividades agropecuarias, dando como resultado una deficiente proporción de renuevos. Se discuten las posibilidades de conservación de la especie in situ y ex situ.

Palabras clave: Pinus greggii, distribución natural, demografía, conservación in situ y ex situ.

\section{ABSTRACT}

Pinus greggii Engelm. is endemic to eastern Mexico and of great ecological and economic importance. It occurs between $20^{\circ} 13^{\prime}$ and $25^{\circ} 29^{\prime} \mathrm{N}$ latitude with a large gap of $360 \mathrm{~km}$ in the central portion of its range. The northern populations of the species are recognized as $P$. greggii var. greggii and the southern ones as $P$. greggii var. australis. The elevational range of the species is from 1,400 to 2,613 m above sea level. Populations in the southern region are located at lower elevations with higher mean temperatures and annual 
precipitation $\left( \pm 1,720 \mathrm{~m}, \pm 17.5^{\circ} \mathrm{C}\right.$ and $\left.\pm 1,370 \mathrm{~mm}\right)$ than populations in the northern region $\left( \pm 2,450 \mathrm{~m}, \pm 13^{\circ} \mathrm{C}\right.$ and $\left.\pm 640 \mathrm{~mm}\right)$. Twenty natural populations of the species were sampled, and huge differences were found among them in the area that they cover and the associated tree species. Southern populations are typically associated with species of temperatesubtropical tree communities, whereas in the northern region tree species from colder and semi-arid communities are more common. Juniperus flaccida and Quercus spp. are common to both regions. In general, populations at the extreme of the natural distribution were smaller and had a lower density of mature trees and seedlings. Most P. greggii populations are subjected to great human impact associated with wood extraction and farming activities, resulting in reduced regeneration. Options for in situ and ex situ conservation of this species are discussed.

Key words: Pinus greggii, natural distribution, demography, in situ and ex situ conservation.

\section{INTRODUCCIÓN}

Pinus greggii Engelm. ex Parl. es una especie endémica de México con gran importancia ecológica y económica. Este pino se distribuye en poblaciones aisladas a lo largo de la Sierra Madre Oriental, en zonas semiáridas y a veces semitropicales. Actualmente se reconocen dos variedades taxonómicas, $P$. greggii var. greggii, que habita la porción norte del área de distribución de la especie, y P. greggii var. australis en el sur (Donahue y López-Upton, 1999), sin traslapes entre ellas. La especie es uno de los componentes estructurales clave en las comunidades en las que crece, ya que forma parte del dosel dominante y en muchos de los casos es la única que representa al estrato arbóreo. Por lo tanto, en los ecosistemas forestales en que se desarrolla, diversas plantas herbáceas y arbustivas dependen del microambiente que genera este árbol.

Por otro lado, a nivel regional, $P$. greggii es uno de los árboles de mayor valor económico para las poblaciones humanas que habitan en zonas aledañas. Se aprovecha para la obtención de madera para la industria del aserrío, y localmente en la obtención de postes para cerca y leña combustible. Además, P. greggii ha mostrado altas tasas de crecimiento en altura y diámetro en ensayos de plantaciones (López et al., 1999; Salazar et al., 1999; Azamar et al., 2000), así como un gran potencial para adaptarse a condiciones limitantes de humedad (Vargas y Muñoz, 1988, 1991; López y Muñoz, 1991). Estas características favorecen el uso de $P$. greggii en programas de reforestación para la recuperación de suelos degradados en diferentes partes de México y en programas de plantaciones comerciales en sitios marginales donde no se adaptan otras especies de Pinus. En México, es la cuarta especie de pino en términos de importancia en plantaciones del Programa Nacional de Reforestación. En Sudáfrica se han establecido plantaciones con P. greggii en 
localidades con niveles bajos de precipitación pluvial, donde $P$. patula Schl. et Cham. y $P$. taeda L., árboles considerados internacionalmente como altamente productivos, no pueden prosperar (Dvorak y Donahue, 1993; Dvorak et al., 1996).

De 1985 a 1995, la Central America \& Mexico Coniferous Resources Cooperative (CAMCORE) de la Universidad Estatal de Carolina del Norte en EUA y el Centro de Genética Forestal A.C. en México, realizaron colectas de semilla de la mayoría de las poblaciones conocidas de $P$. greggii (López-Upton y Donahue, 1995; Donahue y López-Upton, 1996; Dvorak et al., 2000). Exploraciones recientes efectuadas por los autores han permitido la localización de otras poblaciones no registradas. La mayoría de las comunidades vegetales correspondientes se encuentran en proceso de alteración por causas antropocéntricas, originando la fragmentación y el aislamiento de varias poblaciones, mismas que presentan un reducido número de individuos, con riesgo de la permanencia de la especie. El presente estudio tiene como objetivo describir la ubicación geográfica y el estado general de las poblaciones naturales conocidas de Pinus greggii en términos de tamaño y estructura poblacional, con el propósito de establecer las prioridades y lineamientos generales para su conservación in situ y ex situ.

\section{MÉTODOS}

Para la definición de la distribución natural de $P$. greggii se utilizó la información registrada por CAMCORE (Dvorak y Donahue, 1993), por el Colegio de Postgraduados (López et al., 1993; López-Upton y Donahue, 1995; Donahue y López-Upton, 1996), así como la obtenida de ejemplares de herbario y de observaciones efectuadas en áreas intermedias a los sitios conocidos con anterioridad. Con base en las coordenadas geográficas determinadas con un posicionador geográfico Modelo GPIII, se ubicaron en cartas topográficas un total de 20 poblaciones naturales de $P$. greggii, localizadas en los estados de Puebla, Hidalgo, Querétaro, San Luis Potosí, Coahuila y Nuevo León. Se realizaron recorridos para estimar el tamaño en superficie de terreno de cada población, ubicando sus límites en las cartas topográficas con el apoyo del geoposicionador, un altímetro y la observación de los accidentes naturales del terreno (cañadas, elevaciones, valles, etc.). En el laboratorio se obtuvo una copia de la silueta de cada rodal delimitado en las cartas topográficas, cuya superficie se calculó con la ayuda de un planímetro óptico y de un integrador digital de área.

En cada población se levantaron uno a tres sitios de muestreo de $1000 \mathrm{~m}^{2}$ (un total de 56 sitios en las 20 localidades) para obtener información sobre la composición del estrato arbóreo y arbustivo leñoso, así como sobre la estructura de cada una de ellas. Aunque un análisis detallado de esta última podría requerir 
un tamaño de muestra más grande, especialmente en los rodales de mayor extensión, el número de sitios de muestreo utilizado es suficiente para hacer un diagnóstico de la situación general que presentan las poblaciones de la especie en su conjunto, dado el enfoque y la escala regional del estudio. Se cuantificó la densidad de los árboles o arbustos maduros de otras especies para determinar las plantas del estrato superior asociadas naturalmente a $P$. greggii.

En cada sitio se determinó el número de individuos de $P$. greggii, agrupándolos en tres categorías de edad: a) árboles maduros o en edad reproductiva, b) individuos premaduros y c) renuevos. Se consideraron como árboles maduros a los que presentaban conos o indicios de eventos reproductivos anteriores, independientemente de sus dimensiones. Los árboles premaduros fueron aquellos individuos juveniles iguales o mayores de $4 \mathrm{~m}$ de altura en los que no se observaron estróbilos. Se consideraron como renuevos todos los individuos en estado de plántula y juveniles menores de 4 metros de altura. A su vez, esta última categoría se subdividió en tres de acuerdo con su tamaño: renuevos de 1 a $30 \mathrm{~cm}$, de 30 a $100 \mathrm{~cm}$ y de $1 \mathrm{~m}$ hasta $4 \mathrm{~m}$ de altura.

\section{RESULTADOS Y DISCUSIÓN}

Localización y tamaño de las poblaciones de P. greggii

El área de distribución natural conocida de la especie abarca de los $20^{\circ} 13^{\prime}$ hasta a los $25^{\circ} 29^{\prime}$ de latitud norte, una diferencia de un poco más de $5^{\circ}$ entre los sitios más alejados (Fig. 1). En el extremo norte se ubica la población localizada en Puerto El Conejo en los linderos estatales de Coahuila y Nuevo León; en el límite sur se encuentra la ubicada en Patoltecoya, en la región norte de Puebla.

$P$. greggii está en serio peligro de desaparecer en la comarca de Patoltecoya, ya que en la actualidad sólo queda un árbol adulto de la población original en la finca "El Ayacahuite". Sin embargo, el Sr. Juan Ramón Fernández A. ${ }^{\dagger}$, propietario de este predio, realizó una plantación de aproximadamente 10 ha con semilla de los pocos árboles remanentes que constituyeron la población nativa de esta localidad. Aunque se tiene información de los habitantes del lugar de que hay árboles similares en los alrededores, no fue posible ubicarlos. La probable localización en el futuro de otros rodales de $P$. greggii en esta zona sería de gran valor en la conservación de los recursos genéticos de la especie por encontrarse en el extremo sur del área de distribución, y por la situación particular que presenta actualmente la población de Patoltecoya.

En el sentido latitudinal es posible distinguir dos grandes áreas en la distribución natural de la especie, separadas por la discontinuidad geográfica que 


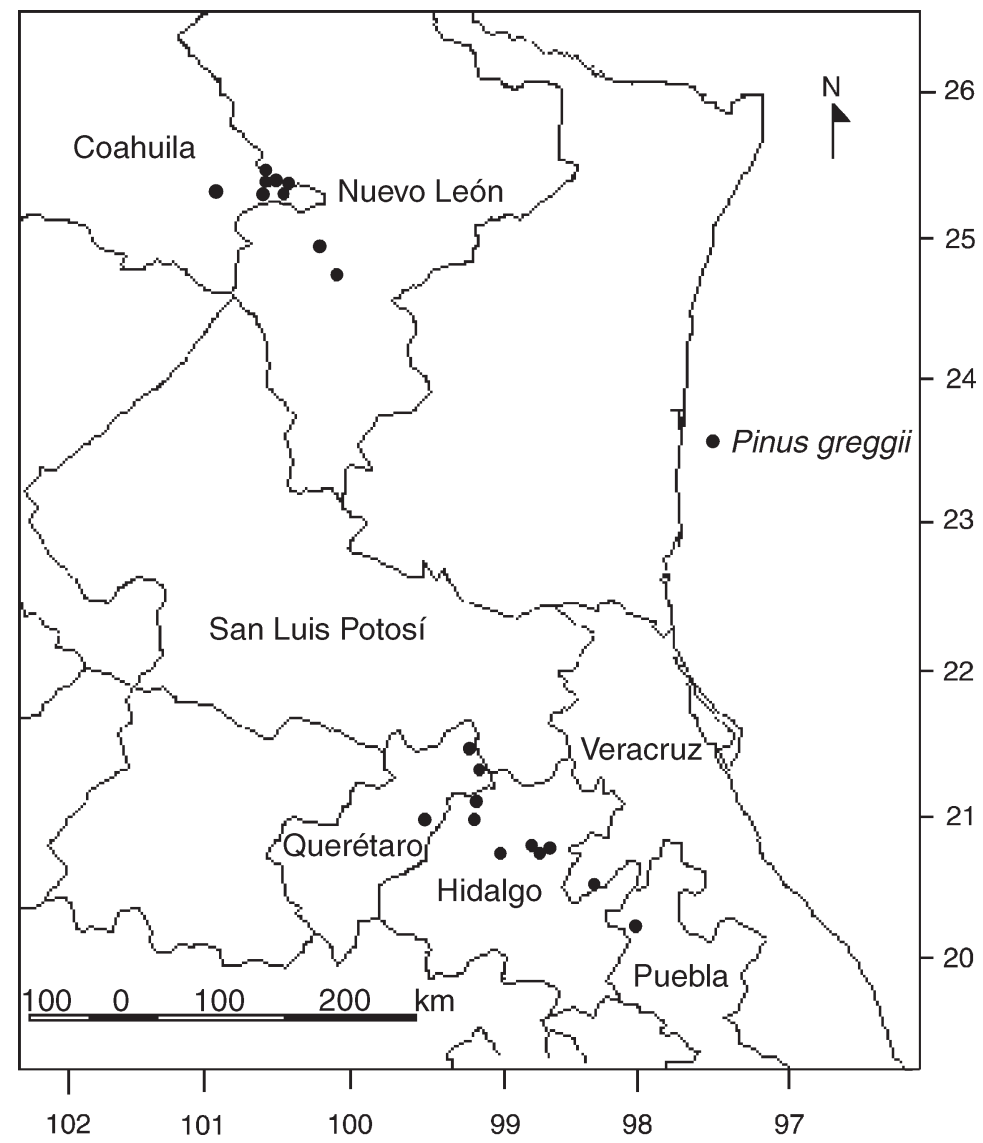

Fig. 1. Distribución de las 20 localidades de $P$. greggii en estudio.

se presenta entre los $21^{\circ} 30^{\prime}$ y $24^{\circ} 30^{\prime}$ de latitud norte (Cuadro 1). La región sur incluye 11 poblaciones conocidas en los estados de Puebla, Veracruz, Hidalgo, Querétaro y San Luis Potosí, todas ellas de la var. australis, mientras que el sector norte incluye nueve poblaciones naturales conocidas de la var. greggii en los estados de Nuevo León y Coahuila (Cuadro 1). Se desconocen las causas de esta disyunción, ya que la Sierra Madre Oriental carece de notable interrupción en el tramo correspondiente, aunque cabe señalar que éste no ha sido explorado completamente debido a lo abrupto de las cordilleras y a la ausencia de vías de comunicación adecuadas. Es posible que futuras búsquedas de campo demuestren que existen otros sitios naturales de $P$. greggii entre $\operatorname{los} 22^{\circ}$ y $24^{\circ} \mathrm{N}$. Recientemente se tiene la información 


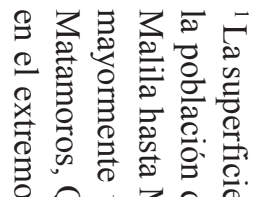
융 훙 के 政

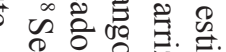
○ 2 党 $\Leftrightarrow \Omega \hat{\circ}$

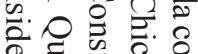

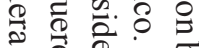
की की ○ き ใै 용 कू ?

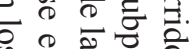
>. बें वै के

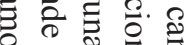
记

$\nabla=\overrightarrow{0} 0$

Е $\cong \Omega$ จ

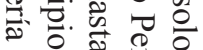

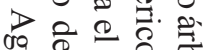

๑ 닐

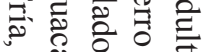
四 坣

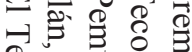
․ 记 茂

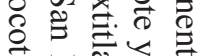
$<$ 능 के

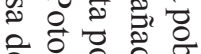

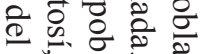

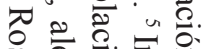

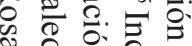

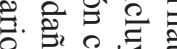
○ ᄋ ¿

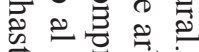
ڤิ) प1 के 붕요

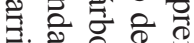
륭ํㅇㅇㅠ

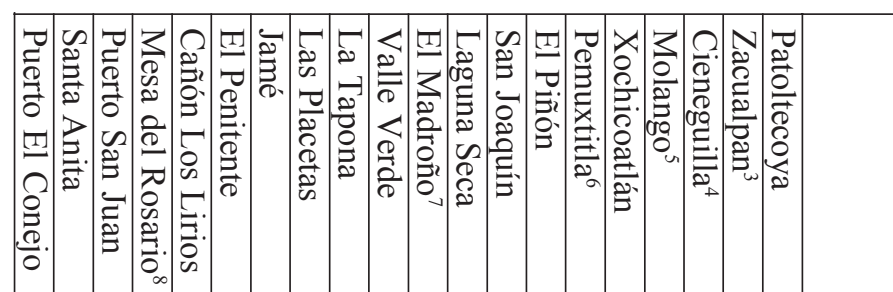

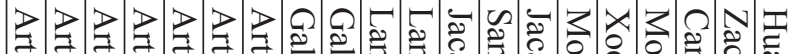

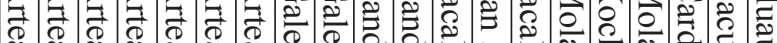
กิ

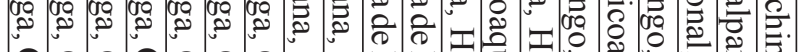

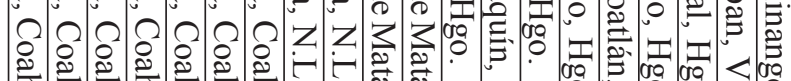

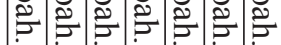

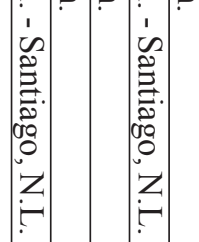

?ִ

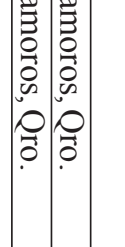

xo:

sa

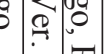

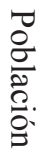

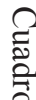

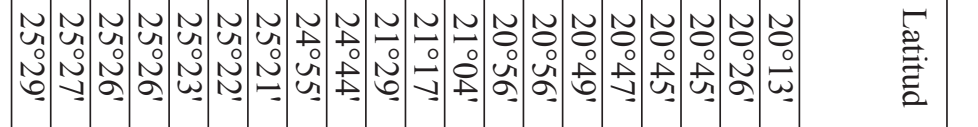

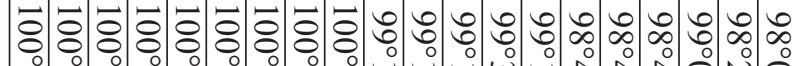

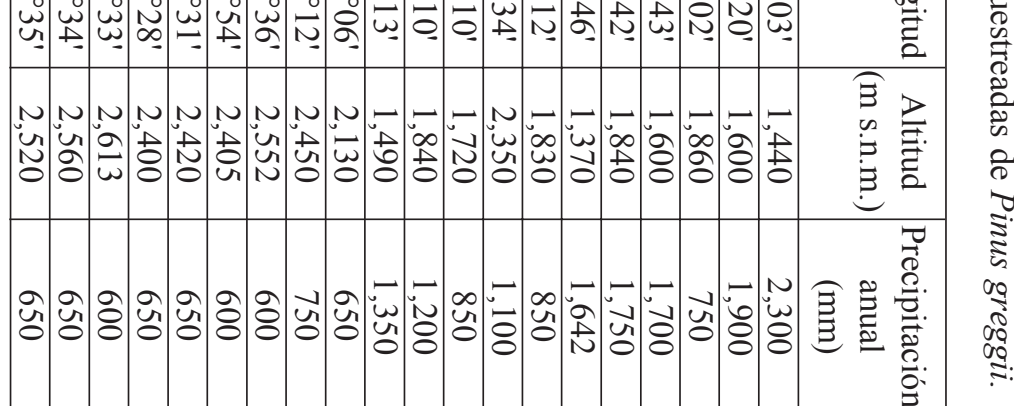

त्व

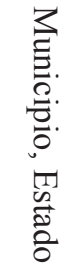
家

(10.0

苛

है

正

\begin{tabular}{|c|c|c|c|c|c|c|c|c|c|c|c|c|c|c|c|c|c|c|}
\hline w & & $\vec{\omega}$ & $\vec{\omega}$ & $\bar{\omega}$ & $\bar{\omega}$ & $\bar{\omega}$ & $\bar{D}$ & $\vec{u}$ & $\Xi$ & $\nabla$ & $\vec{\infty}$ & $\vec{a}=$ & $\omega$ & 二 & $=$ & ज & $\vec{\infty}$ & 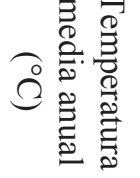 \\
\hline 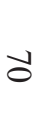 & w & Nu & స్心 & $\begin{array}{l}w \\
\stackrel{0}{0}\end{array}$ & $\stackrel{N}{\circ}$ & $w_{v}$ & $\stackrel{N}{\circ}$ & 그 & $\mid$ & $\begin{array}{l}v \\
u \\
\circ \\
8 \\
8\end{array}$ & 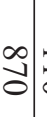 & E) & $\begin{array}{l}0 \\
0 \\
0\end{array}$ & $\vec{N}$ & $\infty$ & Nu & 요 & : \\
\hline
\end{tabular}


de que en el herbario del Instituto de Ecología, A.C. en Pátzcuaro, Michoacán, se encuentra un ejemplar colectado en la Sierra de Catorce, San Luis Potosí, la que se ubica aproximadamente en $23^{\circ} 20^{\prime} \mathrm{N}$.

En lo que respecta a la longitud geográfica, las poblaciones se sitúan desde los $98^{\circ} 03^{\prime} \mathrm{W}$ hasta los $100^{\circ} 54^{\prime} \mathrm{W}$, lo que representa una diferencia de casi $3^{\circ}$. La región de Patoltecoya se localiza en el extremo este de la distribución natural, y la del cerro El Penitente en el extremo oeste (Cuadro 1). Altitudinalmente, la especie se ubica entre los 1,400 y los 2,613 m s.n.m., en un intervalo de más de 1,200 m. En términos generales, las poblaciones de la región meridional habitan áreas situadas también más al este, con menor elevación, así como mayor temperatura media y precitación anual $\left(1,370\right.$ a $2,350 \mathrm{~m}, 16$ a $18^{\circ} \mathrm{C}$ y 750 a $\left.2,300 \mathrm{~mm}\right)$, que las de la región septentrional $\left(2,130\right.$ a $2,613 \mathrm{~m}, 13$ a $15^{\circ} \mathrm{C}$ y 600 a $\left.750 \mathrm{~mm}\right)$. La mayoría de estas últimas están en serio riesgo de perderse, ya que tienen una superficie relativamente pequeña, generalmente menor de 100 ha, con excepción de las poblaciones de Cañón Los Lirios, Mesa del Rosario, La Tapona y Las Placetas, que se extienden sobre más de 150 ha. Durante 1998 el bosque de Las Placetas en el cerro El Potosí se quemó casi en su totalidad. A causa de las condiciones de sequía que prevalecen en la región, es necesario establecer medidas permanentes de protección contra incendios para asegurar la conservación de la especie.

Con excepción de cuatro de ellas, las poblaciones en la región sur tienen superficies mayores de las 300 ha; destacan El Madroño y Valle Verde con un poco más de 5,000 ha (Cuadro 1), por lo que el riesgo de destrucción por un evento catastrófico es reducido. Las de Xochicoatlán, Molango y Pemuxtitla están muy próximas entre sí, por lo que podrían considerarse como una sola población (Cuadro 2). Sin embargo, existen gradientes ambientales que las separan; por ejemplo, hay casi $500 \mathrm{~m}$ de diferencia en altitud entre Pemuxtitla y Xochicoatlán en una distancia de $5 \mathrm{~km}$, lo que influye sobre las condiciones ecológicas y modifica la presencia de especies asociadas (Cuadro 3). Datos de análisis de isoenzimas muestran que existen diferencias entre los árboles de $P$. greggii de estos dos sitios en las frecuencias alélicas de varios loci (Parraguirre et al., 2002).

Las poblaciones del sector norte se encuentran más cercanas entre sí en términos generales, pues con excepción de las distancias entre La Tapona y Las Placetas $(22 \mathrm{~km})$ y entre El Penitente y Jamé $(26 \mathrm{~km})$, ninguna sobrepasa $5 \mathrm{~km}$ (Cuadro 2). En cambio, las poblaciones de la porción sur se localizan a distancias que varían generalmente de 15 a $40 \mathrm{~km}$; sólo las tres ubicadas en los municipios de Molango y Xochicoatlán están separadas entre sí por menos de $15 \mathrm{~km}$.

Dentro de cada región el aislamiento y la presencia de barreras orográficas deben dificultar el libre intercambio de polen, reduciendo la posibilidad del cruzamiento natural entre los rodales separados, especialmente en la var. australis. Sin duda, el intercambio genético entre las dos regiones es muy difícil debido a la separación entre ellas $(360 \mathrm{~km})$. 
Cuadro 2. Distancia entre las poblaciones de Pinus greggii más cercanas entre sí.

\begin{tabular}{|llc|}
\hline Población & Población más cercana & $\begin{array}{c}\text { Distancia } \\
(\mathrm{km})\end{array}$ \\
\hline Patoltecoya & Zacualpan & 40 \\
Zacualpan & Patoltecoya & 40 \\
Cieneguilla & El Piñón & 25 \\
Molango & Xochicoatlán y Pemuxtitla & 5 \\
Xochicoatlán & Molango & 5 \\
Pemuxtitla & Molango & 5 \\
El Piñón & Laguna Seca & 15 \\
San Joaquín & El Piñón & 34 \\
Laguna Seca & El Piñón & 15 \\
El Madroño & Valle Verde & 13 \\
Valle Verde & El Madroño & 13 \\
La Tapona & Las Placetas & 22 \\
Las Placetas & La Tapona & 22 \\
Jamé & Cañón Los Lirios & 5 \\
El Penitente & Jamé & 26 \\
Cañón Los Lirios & Puerto San Juan & 4 \\
Mesa del Rosario & Puerto San Juan & 7 \\
Puerto San Juan & Santa Anita & 3 \\
Santa Anita & Puerto San Juan & 3 \\
Puerto El Conejo & Santa Anita & 4 \\
\hline
\end{tabular}

Especies leñosas asociadas a $P$. greggii

De las diferentes especies leñosas asociadas a Pinus greggii, sólo Juniperus flaccida Schlecht. y Arbutus sp. se encuentran en ambas regiones geográficas (Cuadro 3). Sin embargo, estas dos especies se encuentran ausentes en áreas con precipitaciones mayores a 1,000 $\mathrm{mm}$ anuales y en ambientes de clima más cálido como son las localidades de Patoltecoya, Zacualpan y la zona de MolangoXochicoatlán. El género Quercus está abundantemente representado en la mayoría de las localidades de $P$. greggii, excepto en Pemuxtitla, Xochicoatlán y La Tapona, donde no se registra. Una diferencia notoria entre las regiones sur y norte es que mientras en la primera existen varias especies arbóreas de Quercus asociadas a las 
Cuadro 3. Estimación del número de individuos por hectárea de especies arbóreas y algunas arbustivas asociadas en las 20 poblaciones naturales de Pinus greggii estudiadas.

\begin{tabular}{|c|c|c|c|c|c|c|c|c|c|c|c|c|c|}
\hline Localidad & $\begin{array}{l}\text { Juniperus } \\
\text { flaccida }\end{array}$ & $\begin{array}{c}\text { Quercus } \\
\text { spp. }\end{array}$ & \multicolumn{2}{|c|}{$\begin{array}{c}\text { Alnus } \\
\text { sp. }\end{array}$} & $\begin{array}{l}\text { Arbutus } \\
\text { sp. }\end{array}$ & $\begin{array}{c}\text { Acacia } \\
\text { farnesiana }\end{array}$ & $\begin{array}{c}\text { Pinus } \\
\text { pseudostrobus }\end{array}$ & $\begin{array}{l}\text { Pinus } \\
\text { teocote }\end{array}$ & $\begin{array}{l}\text { Pinus } \\
\text { patula }\end{array}$ & $\begin{array}{c}\text { Pinus } \\
\text { oocarpa }\end{array}$ & \multicolumn{2}{|c|}{$\begin{array}{c}\text { Liquidambar } \\
\text { styraciflua }\end{array}$} & $\begin{array}{c}\text { Tocones } \\
\text { de } \\
\text { árboles }\end{array}$ \\
\hline Patoltecoya & 0 & 0 & \multicolumn{2}{|c|}{0} & 0 & 0 & 60 & 0 & 0 & 0 & \multicolumn{2}{|c|}{0} & 6 \\
\hline Zacualpan & 0 & 180 & \multicolumn{2}{|c|}{0} & 0 & 0 & 0 & 0 & 133 & 0 & \multicolumn{2}{|c|}{33} & 0 \\
\hline Cieneguilla & 150 & 60 & \multicolumn{2}{|c|}{0} & 0 & 160 & 0 & 0 & 0 & 0 & \multicolumn{2}{|c|}{0} & 140 \\
\hline Molango & 0 & 40 & \multicolumn{2}{|c|}{0} & 0 & 0 & 0 & 0 & 0 & 160 & \multicolumn{2}{|c|}{0} & 0 \\
\hline Xochicoatlán & 0 & 0 & \multicolumn{2}{|c|}{0} & 0 & 0 & 0 & 0 & 40 & 0 & \multicolumn{2}{|c|}{0} & 1 \\
\hline Pemuxtitla & 0 & 0 & \multicolumn{2}{|c|}{0} & 0 & 0 & 0 & 0 & 0 & 0 & \multicolumn{2}{|c|}{5} & 0 \\
\hline El Piñón & 257 & 50 & \multicolumn{2}{|c|}{20} & 0 & 0 & 124 & 0 & 0 & 0 & \multicolumn{2}{|c|}{0} & 30 \\
\hline San Joaquín & 87 & 813 & \multicolumn{2}{|c|}{0} & 67 & 0 & 0 & 0 & 0 & 0 & \multicolumn{2}{|c|}{0} & 63 \\
\hline Laguna Seca & 113 & 223 & \multicolumn{2}{|c|}{0} & 10 & 0 & 0 & 10 & 0 & 0 & \multicolumn{2}{|c|}{0} & 257 \\
\hline El Madroño & 517 & 897 & & & 140 & 0 & 0 & 0 & 0 & 0 & & & 103 \\
\hline Valle Verde & 637 & 830 & & & 0 & 0 & 0 & 0 & 0 & 0 & & & 73 \\
\hline Localidad & $\begin{array}{l}\text { Juniperus } \\
\text { flaccida }\end{array}$ & $\begin{array}{c}\text { Quercus } \\
\text { microphylla }\end{array}$ & $\begin{array}{c}\text { Alnus } \\
\text { sp. }\end{array}$ & $\begin{array}{l}\text { Arbutus } \\
\text { sp. }\end{array}$ & $\begin{array}{l}\text { Cupressus } \\
\text { arizonica }\end{array}$ & $\begin{array}{l}\text { Pinus } \\
\text { rudis }\end{array}$ & $\begin{array}{c}\text { Pinus } \\
\text { arizonica }\end{array}$ & $\begin{array}{c}\text { Pinus } \\
\text { cembroides }\end{array}$ & $\begin{array}{l}\text { Pseudotsuga } \\
\text { sp. }\end{array}$ & $\begin{array}{l}\text { Abies } \\
\text { vejarii }\end{array}$ & $\begin{array}{l}\text { Yucca } \\
\text { sp. }\end{array}$ & $\begin{array}{c}\text { Agave } \\
\text { sp. }\end{array}$ & $\begin{array}{c}\text { Tocones } \\
\text { de } \\
\text { árboles }\end{array}$ \\
\hline La Tapona & 17 & 0 & 0 & 0 & 0 & 0 & 0 & 97 & 57 & 0 & 0 & 0 & 3 \\
\hline Las Placetas & 0 & 490 & 0 & 280 & 0 & 0 & 0 & 0 & 53 & 0 & 7 & 0 & 0 \\
\hline Jamé & 190 & 3,800 & 0 & 500 & 0 & 0 & 0 & 0 & 0 & 0 & 10 & 0 & 0 \\
\hline El Penitente & 430 & 3,397 & 0 & 43 & 0 & 0 & 63 & 160 & 7 & 0 & 23 & 0 & 30 \\
\hline Cañón Los Lirios & 50 & 253 & 40 & 0 & 17 & 13 & 7 & 0 & 3 & 273 & 0 & 0 & 17 \\
\hline Mesa del Rosario & 7 & 1,070 & 0 & 150 & 0 & 7 & 0 & 3 & 7 & 0 & 30 & 113 & 0 \\
\hline Puerto San Juan & 105 & 8,235 & 0 & 0 & 0 & 20 & 0 & 125 & 0 & 0 & 0 & 0 & 65 \\
\hline Santa Anita & 0 & 3,590 & 0 & 287 & 0 & 0 & 0 & 0 & 40 & 7 & 0 & 0 & 273 \\
\hline Puerto El Conejo & 40 & 4,860 & 0 & 213 & 27 & 0 & 3 & 70 & 40 & 0 & 0 & 0 & 73 \\
\hline
\end{tabular}


comunidades de $P$. greggii, en la segunda sólo se encuentra $Q$. microphylla Née, un encino enano muy abundante en ciertas áreas de la Sierra de Arteaga, especialmente en Puerto San Juan, con más de 8,000 individuos por ha.

Con menor abundancia se encontraron varias especies de pinos como $P$. patula, P. oocarpa Schiede, P. teocote Schlecht. et Cham. y P. pseudostrobus Lindl. en las poblaciones del sur, así como $P$. rudis Endl., $P$. arizonica Engelm. y $P$. cembroides Zucc. en el norte. Todas las anteriores se encontraron en las áreas de transición de bosque de $P$. greggii a bosques puros de las otras especies, por lo que la abundancia de la planta acompañante varió ampliamente de una localidad a otra. Por ejemplo, P. cembroides se encontró asociado con $P$. greggii en las poblaciones de Mesa del Rosario, Puerto San Juan, Puerto El Conejo, La Tapona y El Penitente, con una densidad que varió desde 3 a 160 árboles por hectárea entre la primera y la última.

En la región norte se encontró Pseudotsuga sp. en 7 de las 9 poblaciones muestreadas, y su densidad varió desde 3 individuos por hectárea en Los Lirios hasta 57 en La Tapona. En esta región se encontraron también plantas típicas de comunidades xerófilas como son Yucca sp. y Agave sp.; y por otra parte algunos individuos de Cupressus arizonica Greene y Abies vejarii Mart. en las zonas de transición a los bosques puros de estas coníferas. En la Sierra de Arteaga se pone de manifiesto un gradiente de comunidades vegetales leñosas conforme aumenta la humedad y la altitud. En un extremo se encuentran las agrupaciones dominadas por Quercus microphylla, seguidas por bosques de P. cembroides, P. greggii, Cupressus arizonica, P. strobiformis Engelm., Abies vejarii y Pseudotsuga sp., hasta Pinus culminicola Andresen \& Beaman en la cima de las sierras.

En nueve de las 20 poblaciones muestreadas de Pinus greggii se encontró una especie del género Arbutus, con una densidad que varió de 10 a 287 individuos/ha. En forma distintiva, en la localidad de Cieneguilla se encontró además Acacia farnesiana (L.) Willd., y en las localidades de Zacualpan y Pemuxtitla se registró Liquidambar styraciflua L., elemento del bosque mesófilo de montaña. En Los Lirios se halló una especie de Alnus que no existe en las otras poblaciones de la región norte.

En el sitio de Zacualpan, donde Pinus greggii se avecina a rodales puros de $P$. patula y $P$. oocarpa, se encontraron árboles con características intermedias entre estos taxa, señal de una posible hibridación natural interespecífica. En este contexto cabe mencionar que Fielding y Nicholson (1956) lograron la hibridación artificial de $P$. greggii y $P$. patula. En la localidad de Pemuxtitla no se registraron especies arbóreas asociadas a $P$. greggii, lo cual posiblemente se debe a la fuerte perturbación ocasionada por actividades agrícolas y ganaderas.

En las poblaciones de Laguna Seca, Cieneguilla y El Madroño se encontró un número abundante de tocones de árboles, evidencia de una perturbación por 
extracción de madera. En las poblaciones de Zacualpan, Molango, Pemuxtitla y Xochicoatlán no se hallaron indicios de extracción de madera, pero la vegetación natural está fuertemente afectada por prácticas agrícolas y pecuarias que han inducido un cambio en el uso del suelo. En el sitio de Xochicoatlán, la mayor parte del arbolado se ubica en áreas con pendientes muy pronunciadas de difícil acceso.

Densidad del arbolado y estructura demográfica

Se encontraron diferencias notorias entre las poblaciones en la estructura demográfica y densidad de arbolado (Cuadro 4). Las de Puerto San Juan y El Penitente en Coahuila, de Molango y Xochicoatlán en Hidalgo, Zacualpan, Ver., y Patoltecoya, Pue. presentaron una densidad menor de 200 individuos por hectárea (incluyendo renuevos, plantas juveniles y adultos). A excepción de la de Puerto San Juan, estas poblaciones se encuentran en los extremos de la distribución natural de la especie, considerando latitud, longitud o altitud. En cambio las de El Madroño, Las Placetas, La Tapona y Mesa del Rosario presentaron una densidad por arriba de los 700 árboles por ha, siendo la última la que registra mayor densidad de plantas adultas.

En las poblaciones de Pemuxtitla, Molango y Xochicoatlán no se encontraron individuos premaduros ni renuevos, pero sí se observaron evidencias de una alta producción de semilla en varios años previos a la realización del presente estudio. En estas localidades es necesario implementar prácticas silvícolas y de protección que permitan el establecimiento de la regeneración para asegurar la permanencia de la especie. En las áreas aledañas a la Laguna Atezca se detectó una repoblación abundante de $P$. greggii en lugares desmontados con fines agrícolas; sin embargo, las posibilidades de establecimiento del arbolado en esos sitios son mínimas, ya que las actividades agropecuarias son permanentes. En el rodal de Laguna Seca no se encontraron individuos juveniles, pero sí una cantidad relativamente elevada de renuevos. Aunque se determinó un número aceptable de árboles maduros por hectárea, estos son de diámetros pequeños, probablemente debido a la tala existente, que aprovecha plantas con diámetros mayores de $35 \mathrm{~cm}$.

Por otro lado, más de $50 \%$ de las poblaciones presentaron una proporción de renuevos/árboles maduros menor de 1, y sólo una de ellas (Cañón Los Lirios) registró más de 2.5 renuevos por cada árbol adulto (Cuadro 4). Una población demográficamente estable requiere de una alta proporción de renuevos para recuperar a los adultos conforme se avanza en las etapas de desarrollo (Ledig et al., 2000). El sitio de Mesa del Rosario, el rodal con mayor densidad de arbolado adulto, también tiene una escasa regeneración natural. Aunque la densidad total de árboles es relativamente elevada, hay una deficiencia general de renuevos, por lo que es conveniente establecer medidas silvícolas para favorecer la repoblación y asegurar la permanencia de la especie. 
Acta Botanica Mexicana 72: 1-16 (2005)

Cuadro 4. Densidad del arbolado (No. de árboles/ha) de diferentes categorías de edad en 20 poblaciones naturales de Pinus greggii.

\begin{tabular}{|c|c|c|c|c|c|c|c|}
\hline \multirow{3}{*}{ Localidad } & \multicolumn{6}{|c|}{ Árboles de Pinus greggii / ha } & \multirow{3}{*}{$\begin{array}{c}\text { Renuevos / } \\
\text { Adultos }\end{array}$} \\
\hline & \multicolumn{3}{|c|}{ Renuevos } & \multirow{2}{*}{ Juveniles } & \multirow{2}{*}{ Adultos } & \multirow{2}{*}{ Total } & \\
\hline & $1-30 \mathrm{~cm}$ & $31-100 \mathrm{~cm}$ & $1-4 \mathrm{~m}$ & & & & \\
\hline Patoltecoya & 0 & 0 & 0 & 0 & 1 & 1 & 0.00 \\
\hline Zacualpan & 5 & 20 & 20 & 48 & 92 & 185 & 0.49 \\
\hline Cieneguilla & 130 & 190 & 110 & 100 & 220 & 750 & 1.95 \\
\hline Molango & 0 & 0 & 0 & 0 & 160 & 160 & 0.00 \\
\hline Xochicoatlán & 0 & 0 & 0 & 0 & 150 & 150 & 0.00 \\
\hline Pemuxtitla & 0 & 0 & 0 & 0 & 210 & 210 & 0.00 \\
\hline El Piñón & 93 & 147 & 143 & 37 & 185 & 605 & 2.07 \\
\hline San Joaquín & 17 & 20 & 47 & 33 & 187 & 304 & 0.45 \\
\hline Laguna Seca & 95 & 126 & 102 & 0 & 217 & 540 & 1.49 \\
\hline El Madroño & 243 & 113 & 173 & 107 & 277 & 913 & 1.91 \\
\hline Valle Verde & 57 & 50 & 140 & 60 & 213 & 520 & 1.16 \\
\hline La Tapona & 213 & 97 & 190 & 23 & 277 & 800 & 1.81 \\
\hline Las Placetas & 220 & 137 & 60 & 40 & 387 & 844 & 1.08 \\
\hline Jamé & 36 & 35 & 44 & 10 & 153 & 278 & 0.75 \\
\hline El Penitente & 0 & 7 & 3 & 0 & 153 & 163 & 0.07 \\
\hline Cañón Los Lirios & 22 & 42 & 256 & 130 & 117 & 567 & 2.74 \\
\hline Mesa del Rosario & 27 & 67 & 100 & 20 & 567 & 781 & 0.34 \\
\hline Puerto San Juan & 30 & 5 & 5 & 0 & 55 & 95 & 0.73 \\
\hline Santa Anita & 10 & 30 & 95 & 0 & 170 & 305 & 0.79 \\
\hline Puerto El Conejo & 56 & 60 & 77 & 3 & 203 & 399 & 0.95 \\
\hline
\end{tabular}


En los sitios de Las Placetas, La Tapona y El Madroño parece no haber problemas de regeneración, ya que existen en cada población más de 500 renuevos por ha en las diferentes categorías de tamaño (Cuadro 4). En esas poblaciones no se encontraron evidencias de pastoreo. Sin embargo, la evaluación de la regeneración en Las Placetas se hizo antes del gran incendio del año 1998 que ocurrió en la zona, por lo que será necesario valorar nuevamente esta región para determinar el grado de recuperación.

En la zona norte, el rodal de El Penitente presenta una baja densidad de repoblación. Esta localidad se encuentra en el extremo oeste del área de distribución natural, relativamente aislada de las otras poblaciones en la Sierra de Arteaga (Cuadro 2). Los sitios de Patoltecoya, Zacualpan, Molango, Pemuxtitla y Xochicoatlán, en el sector meridional, también tienen una densidad baja y escasa regeneración. Estas poblaciones además de haber sufrido un aprovechamiento maderero excesivo, están sometidas a un pastoreo abundante de ganado vacuno. La densidad de la población humana en la zona es alta y la base de su economía es la agricultura y ganadería de subsistencia, por lo que el impacto sobre los ecosistemas forestales es muy elevado.

Conservación in situ y ex situ

La Cooperativa CAMCORE (Dvorak et al., 1996; Dvorak et al., 2000), el Instituto Nacional de Investigaciones Forestales y Agropecuarias (INIFAP), el Centro de Genética Forestal A.C. y el Instituto de Genética Forestal de la Universidad Veracruzana (Alba et al., 1998; Azamar et al., 2000) han recogido semillas de la mayoría de estas poblaciones naturales de Pinus greggii y han realizado ensayos genéticos con fines de producción de germoplasma. Desde 1983 y en años recientes, el personal del Colegio de Postgraduados colectó semilla de todas las poblaciones naturales conocidas de esta especie $\mathrm{y}$ ha iniciado el establecimiento de experimentos en campo con el propósito de evaluar características adaptativas (Salazar et al., 1999; López et al., 2001) e iniciar un programa de conservación ex situ. El programa incluye el acopio de más de 300 lotes de semilla de árboles individuales almacenados en un banco de germoplasma y cuatro ensayos de progenies y procedencias establecidos en diferentes ambientes a lo largo de la distribución natural de la especie. Otras instituciones, como la Universidad Autónoma Agraria Antonio Narro en Coahuila, Protectora de Bosques (PROBOSQUE) del Estado de México, la Comisión Forestal en Michoacán, y el Instituto Nacional de Investigaciones Forestales y Agropecuarias (INIFAP) en Oaxaca y Durango, están efectuando otras pruebas con $P$. greggii, algunas convertidas ya en huertos semilleros para programas de reforestación fuera del área de su distribución natural. También se han establecido importantes ensayos en 
varios países como son Colombia, Chile, Brasil, Zimbabwe y Sudáfrica, con base en materiales procedentes de poblaciones de las dos regiones, así como un proyecto encaminado a la obtención de semilla mejorada en el último país (Dvorak et al., 2000).

Sin embargo, a pesar de que los esfuerzos de colecta de semilla y establecimiento de experimentos con Pinus greggii han sido de los más destacados en México, no existe un programa integrado de conservación a nivel nacional. Se trata más bien de intentos particulares de producción de germoplasma para abastecer programas locales de plantaciones y no con el propósito estricto de conservación ex situ. Existe un movimiento sin control de la semilla del pino, que no contempla las diferencias ambientales entre el origen y el destino del germoplasma, lo que puede generar problemas de mala adaptación de las plantas y fracaso de las plantaciones. Igualmente, se ha realizado una selección fenotípica inadecuada de los progenitores para obtener semilla, lo que genera que aquellas reforestaciones con fines de restauración en terrenos forestales y en las carreteras del centro del país, y especialmente aquellas comerciales, que incluyen las plantaciones de árboles de navidad en el estado de Durango, no sean exitosas por producir individuos de mala calidad. Por otro lado, todas estas plantaciones no se han establecido en áreas naturales de la especie y son pocos los experimentos establecidos en áreas naturales de $P$. greggii, por lo que no representan una fuente de contaminación genética para las poblaciones nativas. Sin embargo, realizar plantaciones con semillas provenientes de localidades con ambientes similares podría ser útil para aumentar la diversidad genética dentro de las poblaciones nativas, la que se ha encontrado que es relativamente reducida (Parraguirre et al., 2002).

Una estrategia adecuada de conservación de recursos genéticos forestales requiere, además de las medidas de conservación ex situ, del manejo apropiado de las poblaciones en su hábitat nativo (Rogers y Ledig, 1996; Ledig et al., 1998). Esta es la manera de asegurar la continuidad del proceso evolutivo de la especie de interés en su área de distribución natural. La importancia de las medidas de conservación in situ de Pinus greggii aumenta si se considera la diversidad de especies arbóreas, arbustivas y herbáceas asociadas y el grado de diversidad ecológica y genética existente en estas comunidades. Utilizando una muestra de 16 loci isoenzimáticos, Parraguirre et al. (2002) encontraron un considerable grado de diferenciación genética entre las poblaciones de $P$. greggii, con un elevado porcentaje de alelos raros y privados. Debido a las posibilidades de pérdida de este tipo de alelos durante el proceso de muestreo para la conservación ex situ (Namkoong, 1988), la mejor opción es la conservación in situ de las poblaciones. Sin embargo, ante la imposibilidad práctica de manejo adecuado de todas las poblaciones naturales, es necesario establecer prioridades con base en diferentes criterios. Así, rodales como los de La Tapona, Los Lirios, Molango, Laguna Seca, El Madroño y Valle Verde son prioritarios por su capacidad de crecimiento en 
ambientes específicos (e.g. Dvorak et al. 2000), el nivel de variación intrapoblacional y las amenazas que enfrentan. Las tres primeras son además relativamente pequeñas, por lo que requieren medidas de conservación adicionales.

\section{AGRADECIMIENTOS}

Se dan las gracias al Fondo Mexicano para la Conservación de la Naturaleza A.C. y al Consejo Nacional de Ciencia y Tecnología por el apoyo financiero proporcionado para llevar a cabo el proyecto de investigación del cual se derivó esta información, a través de los convenios B1-73 y P220-CCOR-904153, respectivamente.

\section{LITERATURA CITADA}

Alba L., J., L. Mendizábal H. y A. Aparicio R. 1998. Respuesta de un ensayo de procedencias de Pinus greggii Engelm. en Coatepec, Veracruz, México. Forestal Veracruzana 1(1): 25-28.

Azamar O., M., J. López U., J. J. Vargas H., y A. Plancarte B. 2000. Evaluación de un ensayo de procedencias-progenies de Pinus greggii y su conversión a huerto semillero. Memorias del 1er. Congreso Nacional de Reforestación. Programa Nacional de Reforestación-Colegio de Postgraduados. Montecillo, Méx. 7 pp.

Donahue, J. K. y J. López-Upton. 1996. Geographic variation in leaf, cone and seed morphology of Pinus greggii Engelm. in native forest. For. Ecol. Manag. 82: 145157.

Donahue, J. K. y J. López-Upton. 1999. A new variety of Pinus greggii (Pinaceae) in Mexico. Sida 18(4): 1083-1093.

Dvorak, W. S. y J. K. Donahue, 1993. Reseña de investigaciones de la cooperativa CAMCORE 1980-1992. Central America \& Mexico Coniferous Resources Cooperative. Raleigh, NC. 94 pp.

Dvorak, W. S., J. E. Kietzka y J. K. Donahue. 1996. Three-year survival and growth of provenances of Pinus greggii in the tropics. For. Ecol. Manag. 83: 123-131.

Dvorak, W. S., J. E. Kietzka, J. K. Donahue, G. R. Hodge y T. K. Stanger. 2000. Pinus greggii. In: Conservation \& Testing of Tropical \& Subtropical Forest Tree Species by the CAMCORE Cooperative. Central America \& Mexico Coniferous Resources Cooperative, North Carolina State University, Raleigh, NC. pp. 52-73.

Fielding, J. M. y D. I. Nicholson. 1956. A hybrid between Pinus patula and Pinus greggii. Austral. Forest. 20: 104-105.

Ledig, F. T., J. J. Vargas H. y K. H. Johnsen. 1998. The conservation of forest genetic resources; case histories from Canada, Mexico, and the United States. J. For. 96: 3241. 
Ledig, F. T., M. Mápula-Larreta y B. Bermejo-Velázquez. 2000. Locations of endangered spruce populations in México and the demography of Picea chihuahuana. Madroño 47(2): 71-88.

López A., J. L., J. J. Vargas H., C. Ramírez H. y J. López U. 1999. Variación intraespecífica en el patrón de crecimiento del brote terminal de Pinus greggii Engelm. Revista Chapingo, Serie Ciencias Forestales y del Ambiente 5(2): 133-140.

López U., J. y A. Muñoz O. 1991. Selección familiar por tolerancia a sequía en Pinus greggii Engelm. I. Evaluación en plántula. Agrociencia, Serie Fitociencia 2(2): 111-123.

López U., J., J. Jasso M., J. J. Vargas H. y J. C. Ayala S. 1993. Variación de características morfológicas en conos y semillas de Pinus greggii Engelm. Agrociencia, Serie Recursos Naturales Renovables 3(1): 81-95.

López U., J., C. Ramírez H., F. O. Plascencia E. y R. Rodríguez L. 2001. Variación intraespecífica en el crecimiento de Pinus greggii. Memorias del 2do. Simposium Internacional Sobre Recursos Naturales Bosque-Suelo-Atmósfera. Jalapa, Ver. pp. 82-83.

López-Upton., J. y J. K. Donahue. 1995. Seed production of Pinus greggii in natural stands in México. Tree Planters' Notes 46(3): 86-92.

Namkoong, G. 1988. Sampling for germoplasm collections. HortScience 23(1): 79-81.

Parraguirre L., C., J. J. Vargas H., P. Ramírez V., H. S. Azpíroz y J. Jasso M. 2002. Estructura de la diversidad genética en poblaciones naturales de Pinus greggii Engelm. Fitotecnia Mexicana 25(3): 279-287.

Rogers, D. L. y F. T. Ledig 1996. The status of temperate North American forest genetic resources. Report 16. University of California Genetic Resources Conservation Program. Davis, California. 85 pp.

Salazar G., G. J., J. J. Vargas H., J. Jasso M., J. D. Molina G., C. Ramírez H. y J. López U. 1999. Variación en el patrón de crecimiento en altura de cuatro especies de Pinus en edades tempranas. Madera y Bosques 5(2): 19-34.

Vargas H., J. J. y A. Muñoz O. 1988. Resistencia a sequía: II. Crecimiento y supervivencia en plántulas de cuatro especies de Pinus. Agrociencia 72: 197-208.

Vargas H., J. J. y A. Muñoz O. 1991. Potencial hídrico, transpiración y resistencia estomatal en plántulas de cuatro especies de Pinus. Agrociencia, Serie Recursos Naturales Renovables 1(3): 25-38. 